\title{
Acute Wound with Varicose Vein in Rural Setting: The Challenge and Importance of Comorbidity Management
}

\author{
Agustini Song, $M D^{1^{*}}$, Kevin Leonard Suryadinata, $M D^{2}$ and Nabila Viera Yovita, $M D^{3}$ \\ ${ }^{1}$ Department of Emergency, S.K. Lerik Public General Hospital, Indonesia \\ ${ }^{2}$ Division of Plastic, Reconstructive, and Aesthetic Surgery, Department of Surgery, Dr. Hasan Sadikin General \\ Hospital/Faculty of Medicine, Universitas Padjadjaran, Indonesia \\ ${ }^{3}$ Department of Physical Medicine and Rehabilitation, Dr. Cipto Mangunkusumo National General Hospital/ \\ Faculty of Medicine, Universitas Indonesia, Indonesia
}

*Corresponding author: Agustini Song, MD, Department of Emergency, S.K. Lerik Public General Hospital, Apartment CBD Pluit Tower Papyrus 10G, Jl. Pluit Selatan Raya, Kel. Penjaringan, Kec. Penjaringan, Jakarta Utara, DKI Jakarta, 14440, Indonesia, Tel: +6281284236333

\begin{abstract}
Background: Acute wound healing with comorbidities, such as a varicose vein, may be impaired and result in prolonged healing and unsightly scar if comorbidity identification and management are not done properly. We aimed to present the alternative comorbidity diagnosis and treatment for better outcome in rural setting.

Case description: A 59-year-old woman was admitted to the ED with an open wound on the dorsolateral side of her left foot with comorbidity of uncontrolled hypertension and varicose vein. After wound closure with simple suture, the Perthes and Brodie-Trendelenburg tests were done which indicated insufficiency of perforator vein grade C3. Elastic bandage and physical activity restriction along with routine wound care and hypertension treatment were administrated with attention on wound exudate and pain level. On the $14^{\text {th }}$ day, a day after the patient had prolonged standing, the wound was heavily macerated; it resolved to secondary healing and achieved full closure on the $53^{\text {rd }}$ day with normal physiologic function and esthetically acceptable scar.

Conclusion: The challenges we encountered in this case consisted of the limitation in diagnostic modality, the patient's non-adherence to the treatment protocol, and the lack of wound dressing choice due to limitations in the rural setting. The combination of excellent wound care, comorbidity management supported by appropriate diagnostic and treatment modality, and patient's adherence to treatment protocol are crucial for functional and esthetic wound healing. Despite challenges in a rural setting, achieving optimal wound healing may still be possible by utilizing alternative diagnostic and treatment choices, together with effective education.
\end{abstract}

\section{Keywords}

Lower extremity wound, Wound management, Wound assessment, Varicose vein

\section{Introduction}

Acute open wound is the third most common injury of emergency department (ED) visits in Indonesia, constituting $23.2 \%$ of all types of injury [1]. Most acute wounds would heal without any difficulty regardless of the cause, but in the presence of local or systemic factors which can hinder wound healing, an acute wound may become chronic. The progression to a chronic wound can be prevented by identification and management of those factors along with appropriate wound management [2]. Failure to identify these problems would prolong the healing process and increase emotional and financial burden for patients, patient's families, and the society [3].

Varicose vein is part of the chronic venous disease spectrum, and it affects approximately $10 \%$ to $30 \%$ of the general population, with higher prevalence in women [4]. Risk factors for varicose vein can be hormonal, lifestyle, inherited, and acquired; some of those are female sex, prolonged standing, obesity, family history, age, and pregnancy [5]. The pathophysiology of varicose 
vein involves venous hypertension, venous valvular incompetence, changes in the vein wall structure, and alterations in shear stress [6]. The degree of varicose vein is categorized using Clinical, Etiology, Anatomy, and Pathophysiology (CEAP) classification, with higher CEAP class indicates more advanced chronic venous disease [7]. The signs and symptoms of varicose veins vary depending on the severity; the patient may feel from asymptomatic to slight discomfort and finally leg heaviness and cramping, and the skin of the affected leg can appear as dry irritated skin in the early stage, to hyperpigmentation, edema, lipodermatosclerosis, and ultimately ulceration $[5,8]$. These changes in skin condition may impair wound healing which leads to the progression of the acute wound into a chronic one.

Given the risk of wound healing impairment by varicose vein, the identification and management of varicose vein as a comorbidity in acute wound patients are mandatory, but these are challenging to conduct in a rural setting with limited diagnostic and treatment modality. We aimed to present and discuss the management of a case with such limitation as a reference for any ED physicians who face similar challenges in their patients.

\section{Case Description}

A 59-year-old woman was admitted to the ED with an open wound on the dorsolateral side of her left foot (Figure 1), with uncontrolled hypertension and a varicose vein on the affected leg (Figure 2). The clean, $3.5 \mathrm{~cm}$-long, subcutaneous-depth wound was sutured, and dressed with paraffin gauze and few layers of sterile gauze, secured with transparent dressing. The Perthes and Brodie-Trendelenburg tests done to confirm the diagnosis of varicose vein suggested insufficiency of perforator vein grade C3 according to the CEAP classification system. An elastic bandage was bound around the leg at the height of the wound and leg elevation while resting and sleeping was advised. Routine wound cares in an outpatient setting every two to three days, along with

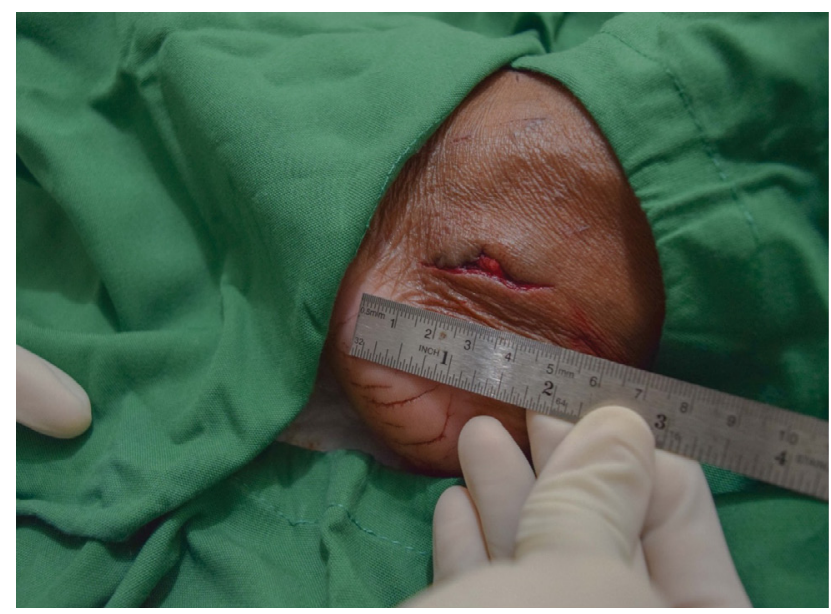

Figure 1: The appearance of the open wound on the dorsolateral side of the left foot. the treatment for grade 1 hypertension and varicose vein with measurement of the exudate level and pain level were performed (Figure 3 ).

On the $14^{\text {th }}$ day, the dressing was saturated along the heavily macerated wound, increased pain level, and edema of the affected leg. The patient admitted standing for four hours straight the day before. All sutures were removed and slough was debrided; the patient was advised to restrict overusing the affected leg, elevate it while resting, and dressing change frequency was increased to daily. Improvements were apparent from the decrease of exudate, decrease of wound size and depth, pain reduction and the absence of infection. Complete wound closure was finally achieved on the $53^{\text {rd }}$ day ( $8^{\text {th }}$ week) with normal physiologic function and esthetically acceptable scar (Figure 4).

\section{Conclusions}

The identification and management of varicose vein as the comorbidity were conducted early since the patient was treated in the ED for her acute wound. After wound management and closure, further history taking, clinical examination, and documentation were done based on early suspicion of the presence of vascular

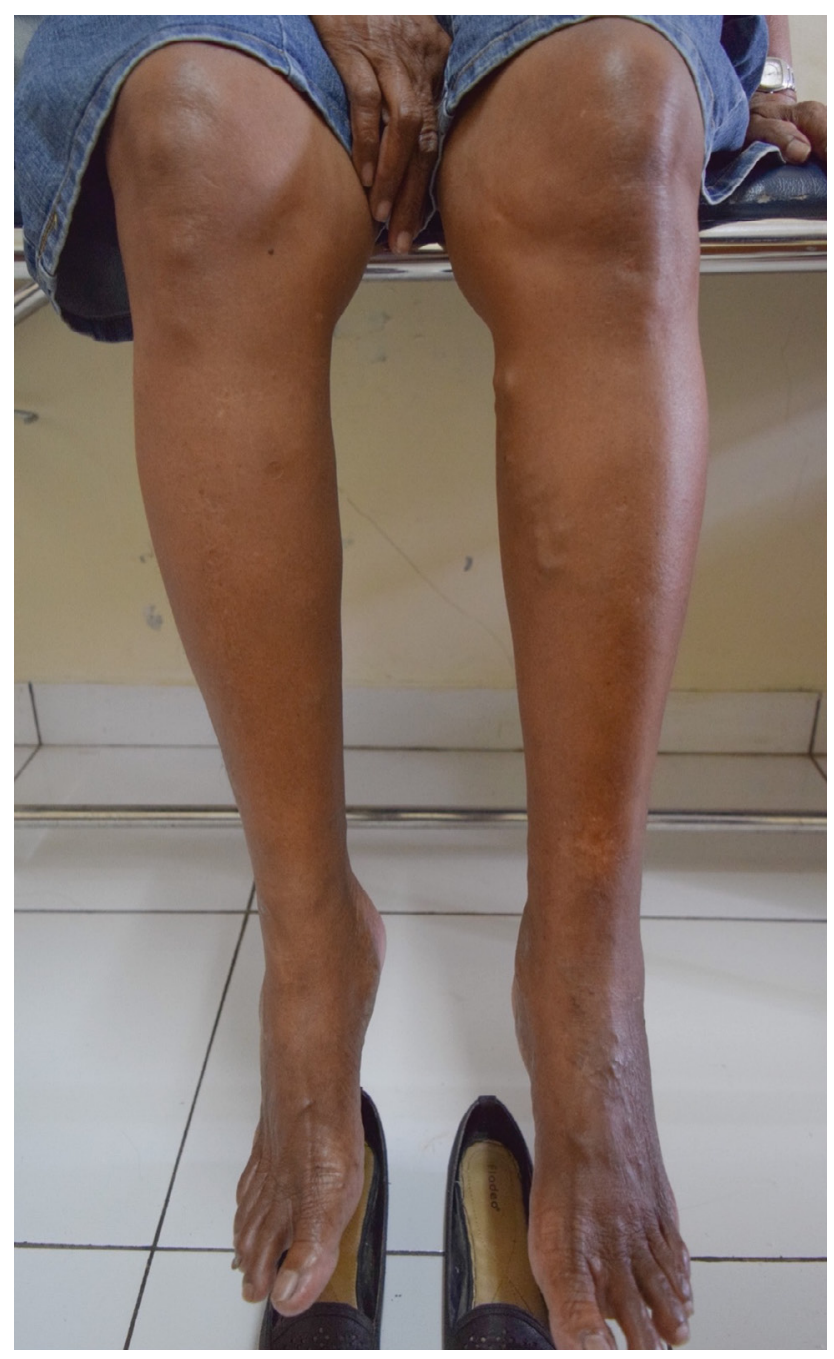

Figure 2: The appearance of varicose vein, more prominent on the left leg. 


\section{Exudate and Pain Level Measurement}

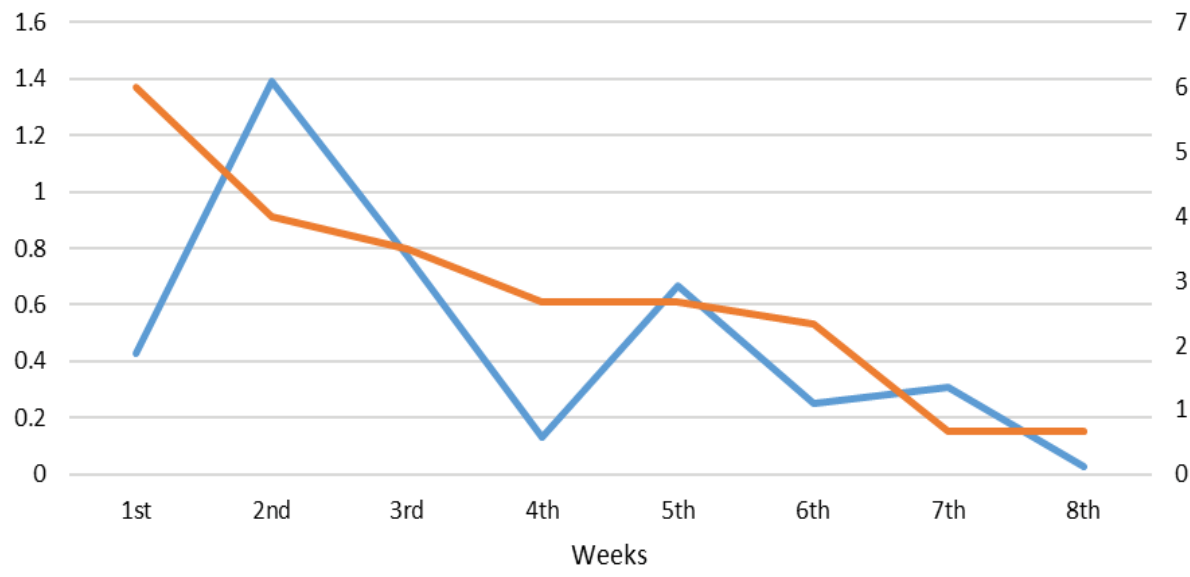

Exudate (grams) Pain (NRS 0-10)

Figure 3: The mean value of exudate and pain level of each week until complete wound healing.

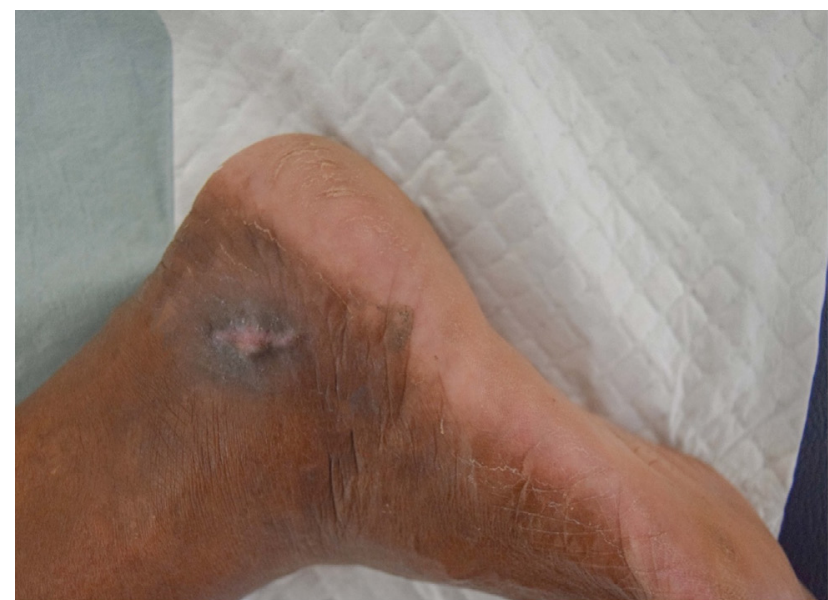

Figure 4: The appearance of the completely healed wound on the $53^{\text {rd }}$ day $\left(8^{\text {th }}\right.$ week).

disorder comorbidity i.e., varicose vein which could defer wound healing. There was no available guideline yet about acute wound management with varicose vein as comorbidity, hence the treatment given was based on consultation with the attending surgeon.

The first challenge was the limitation in diagnostic modality. To confirm the diagnosis of varicose vein and identify the underlying cause, Duplex ultrasound is considered as the gold standard [5,8-12] but because it was not available in our facility, the BrodieTrendelenburg test and the Perthes test were done instead. The Brodie-Trendelenburg test can help to detect venous incompetence and distinguish between superficial venous and deep venous insufficiency $[5,10]$. This test can be performed by a general practitioner (GP) in an ED setting with $91 \%$ sensitivity for the identification of superficial and perforator reflux (91\%), but only $15 \%$ specificity [11]. The Perthes test is used for differentiating between deep venous insufficiency and obstruction, and is feasible for ED setting and GP $[5,10]$ but is also poorly specific $(20 \%)$ regardless of its high sensitivity (97\%) [11]. A study found Duplex ultrasound to be $82.93 \%$ sensitive and $97.56 \%$ specific in the evaluation of perforator competency [13], hence it is a better choice of diagnostic tool, but we suggest that in the absence of Duplex ultrasound, BrodieTrendelenburg test and Perthes test can be beneficial for early detection and management of varicose vein due to their high sensitivity.

The second challenge was the patient's nonadherence to the treatment protocol. Even though problematic wound healing was expected due to the patient's comorbidity, the patient's non-adherence to the medical advice might have contributed to prolonged healing. Despite wound maceration on the $10^{\text {th }}$ day, all sutures were intact and the wound might still have the chance to heal in the acute wound healing timeframe. However, on the $14^{\text {th }}$ day, a day following the day patient stood for four hours straight, the wound and varicose vein condition worsened, which ended with the wound had to resolve by secondary intention and undergo prolonged healing. Recent studies suggested the association between prolonged standing and development of varicose vein [14,15], and the suggested hypothesis regarding this phenomenon was the increase of intravascular hydrostatic pressure in an upright position causing the impeded blood flow and consecutive stasis in veins of the lower extremities. Because stasis in the venous system is a key mechanism in venous vascular disease, therefore prolonged standing may lead to venous vascular disease [15]. In our case, the prolonged standing might reasonably worsen the existing varicose vein in the form of leg edema and increasing pain level. The fluid accumulation due to edema might contribute to more wound exudate and maceration which impeded the wound healing process. Increasing the patient's adherence to treatment protocol resulted in improvement of the wound and varicose vein condition and eventually wound closure, 
which further supported the importance of patient's adherence for optimal treatment outcome.

The third challenge encountered was the lack of wound dressing choice due to limitations in rural setting. As the wound passed its normal timeframe of wound healing (10 to 14 days according to The American Academy of Family Physician guideline) [16], re-evaluation and adjustment of wound dressing were supposed to be conducted. As the wound, in this case, was highly exudative and macerated, dressings with powerful absorptive capacities which also reduce maceration of surrounding skin (such as alginates, hydrofibers, foams, and polymeric membrane dressings) would be appropriate [17]. Unfortunately, the available dressing in our facility was limited to paraffin gauze and additional cost for wound dressing would be burdensome for the patient. Therefore, the adjustment possible was only by increasing the frequency of dressing change to manage wound exudate and reduce maceration. This absence of proper wound dressing might contribute to slow healing of this wound.

Wound healing in a patient with comorbidity depends heavily on comorbidity management along with appropriate wound care. The importance of comorbidity management cannot be ruled out, as excellent wound care alone would not be sufficient for optimal wound healing in these patients. In the rural setting, limitation in wound dressing choice and comorbidity diagnosis and treatment modality, worsened by the patient's incompliance in many cases, are what many physicians find challenging in handling such cases. Nevertheless, optimal wound healing may still be achieved by utilizing alternative diagnostic and treatment choices, together with effective patient's education, with the intention to achieve optimal wound healing, both functionally and esthetically. Wound management in a patient with comorbidity should therefore, involve the combination of excellent wound care, comorbidity management supported by appropriate diagnostic and treatment modality, and patient's adherence to treatment protocol.

\section{Acknowledgement}

None.

\section{Conflict of Interest}

The authors declare that there is no conflict of interest.

\section{Authors Contribution}

All authors have contributed equally.

\section{References}

1. Trihono (2013) Badan penelitian dan pengembangan kesehatan kementerian kesehatan RI. Riset Kesehatan Dasar: 101-104.

2. Grey JE, Enoch S, Harding KG (2006) ABC of wound healing: Wound assessment. BMJ 332: 060398.

3. Lazarus GS, Cooper DM, Knighton DR, Margolis DJ, Pecoraro RE, et al. (1994) Definitions and guidelines for assessment of wounds and evaluation of healing. Arch Dermatol 130: 489-493.

4. Evans CJ, Fowkes FGR, Ruckley CV, Lee AJ (1999) Prevalence of varicose veins and chronic venous insufficiency in men and women in the general population: Edinburgh vein study. J Epidemiol Community Health 53: 149-153.

5. Piazza G (2014) Varicose veins. Circulation 130: 582-587.

6. Bergan JJ, Schmid-Schönbein GW, Smith PDC, Nicolaides AN, Boisseau MR, et al. (2006) Chronic venous disease. N Engl J Med 355: 488-498.

7. Lurie F, Passman M, Meisner M, Dalsing M, Masuda E, et al. (2020) The 2020 update of the ceap classification system and reporting standards. J Vasc Surg Venous Lymphat Disord 8: 342-352.

8. Hamdan A (2012) Management of varicose veins and venous insufficiency. JAMA 308: 2612-2621.

9. Lin F, Zhang S, Sun Y, Ren S, Liu P (2015) The management of varicose veins. Int Surg 100: 185-189.

10. Krishnan S, Nicholls SC (2005) Chronic venous insufficiency: Clinical assessment and patient selection. Semin intervent Radiol 22: 169-177.

11. Kim J, Richards S, Kent PJ (2000) Clinical examination of varicose veins - a validation study. Ann R Coll Surg Engl 82: 171-175.

12. Eberhardt RT, Raffetto JD (2014) Chronic venous insufficiency. Circulation 130: 333-346.

13. Prabhu MA, Natarajan KG, Smile S (2017) Comparison of clinical tests and doppler ultrasound in the preoperative assessment of lower extremity varicose veins. Indian $\mathrm{J}$ Vasc Endovasc Surg 4:51.

14. Bahk JW, Kim H, Jung-Choi K, Chul Jung M, Lee I (2012) Relationship between prolonged standing and symptoms of varicose veins and nocturnal leg cramps among women and men. Ergonomics 55: 133-139.

15. Tuchsen F, Hannerz H, Burr H, Krause N (2005) Prolonged standing at work and hospitalisation due to varicose veins: A 12 year prospective study of the danish population. Occup Environ Med 62: 847-850.

16. Forsch RT, Little SH, Williams C (2017) Laceration repair: A practical approach. Am Fam Physician 95: 628-636.

17. Dabiri G, Damstetter E, Phillips T (2016) Choosing a wound dressing based on common wound characteristics. Advances in Wound Care 5: 32-41. 